\title{
Genomics separates the sheep from the goats
}

Sheep were the first grazing animals bred to accentuate desirable traits, such as the production of meat, wool and milk. Their domestication began 9,000 years ago in northern Iraq and nearby areas in Iran. The extraordinary abilities of sheep to adapt to nutritionally poor diets and climatic extremes led to the spread of sheep husbandry across the globe, generating an unusually rich variety of phenotypes among today's many breeds. The recent report in Science of a high-quality, 2.61-Gb reference sheep genome ${ }^{1}$, assembled by the analysis of two individuals of the Texel breed, highlights the genetic differences between sheep and other animals and will help to identify targets for the genetic improvement of sheep.

Comparative genomic analysis suggests that sheep became a distinct species from goats $\sim 4$ million years ago. Cattle, yaks, pigs, camels and horses are progressively more distant relatives of sheep. The genome sequence, together with transcriptomic analysis of 94 samples from 40 different tissues, including 83 samples from four Texel sheep other than the two sequenced, provide important clues as to how sheep process lignocellulose-rich plant material into animal protein and produce wool. The most striking insights into both processes come from comparison of genes that control development of the rumen and the skin.

The rumen is the first division of the four-compartment 'stomach', in which most food collects immediately after being swallowed and from which it is later returned to the mouth as cud for thorough chewing. A tough keratinized lining, which is similar to the skin, distinguishes the rumen from the true stomach. Elisha Gootwine of the Volcani Center in Bet Dagan, Israel, notes the importance of the rumen in the evolution of traits. "Rumen function is probably a limiting factor for many traits related to the growth and reproduction of a host of farm animals," he says. "But owing to the complex role played by microbiota in the efficiency of food conversion and the emission of methane, genetic analysis of rumen efficiency is extremely challenging."

The study provides insights into the genetic basis of the rumen. It reveals that although many genes that are normally expressed in the skin are highly expressed in the rumen, the gene that encodes Trichohyalin-like2 (TCHHL2) and several genes that belong to the $P R D-S P R R / /$ family appear to be uniquely expressed in the wall of the rumen of sheep and cattle. These genes probably contribute to the keratinization of the rumen lining. "I am hopeful that the identification of genes implicated in rumen evolution might open the way for us to select desirable genotypes to maximize the conversion of forage into animal protein, and to minimize environmentally harmful methane emission," says Gootwine.

Tad Sonstegard of the US Department of Agriculture, Agriculture Research Service points to the value of the genome for identifying genes with a strong potential to affect the production of meat and wool in sheep. Fatty acid production has a key role in the synthesis of wool, with the waxy substance lanolin contributing as much as one-quarter of the weight of wool. Unlike TCHHL2, the late cornified envelope 7A ( $L C E 7 A)$ gene, which is expressed in the skins of sheep, cattle and goat, is not expressed in the rumen walls of these animals. The consistent selection of $\angle C E 7 A$ during the breeding of many sheep varieties and the demonstration that $L C E 7 A$ is expressed in wool follicles suggest that it might be involved in wool production. Members of the MOGAT gene family, which encode enzymes that convert monoacylglycerides into diacylglycerides and triacylglycerides, may also contribute to wool production ${ }^{1}$. In humans, MOGAT3 is a single-copy gene that is highly expressed in the human liver and small intestine, where it is involved in the absorption of dietary fat. By contrast, both MOGAT2 and MOGAT3 have undergone tandem expansion in sheep, with none of the copies of MOGAT2 expressed in the liver and one copy of MOGAT2 and three copies of MOGAT3 expressed at high levels in the skin. The LCE7A, MOGAT2 and MOGAT3 genes are thus likely to control wool production.

Although there might be an interest in using these insights to enhance wool production, given the decreases in wool prices over recent years, the need to shear sheep has become more of a nuisance than a boon for many farmers. "Today, meat production is of far greater importance than wool in most parts of the world," explains Sonstegard. "In fact, the removal of wool management

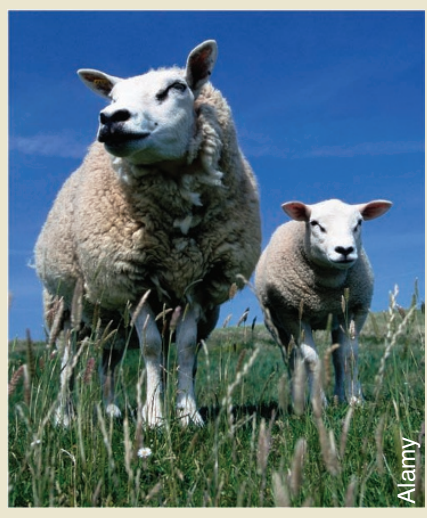

can offer such huge savings in time and labor that sheep that are self-shearing are increasingly in vogue. Given that sheep very probably shed their coats before they were domesticated, and that humans selected for wool retention, it would be useful to understand how to reverse that process without compromising desirable traits."

Some initial insights into the genetic control of traits such as growth rates, meat quality, disease resistance, litter size, lamb survival rates and year-round reproduction have emerged through decades of analysis of sheep quantitative trait loci (QTLs). Runlin Ma, from the Chinese Academy of Sciences in Beijing, emphasizes the potential utility of the new genome sequence for understanding the molecular bases of important phenotypes linked to QTLs. "We need to know what a QTL is before we can use it effectively," he explains. Ma is convinced that many of the frustrations associated with marker-assisted selection over the past few decades might have been avoided if livestock breeders had a better understanding of the genome structures of important farm animals. "I am confident that comparison of genome sequences from sheep breeds from geographically distinct regions will enable us to establish whether a QTL represents a coding region, a promoter, an enhancer or suppressor, or perhaps a small noncoding RNA," he says. "That level of understanding will help considerably to dissect the interactions between QTLs."

Peter Hare, Senior Editor, Nature Biotechnology

1. Jiang, Y. et al. Science 344, 1168-1173 (2014) 